\title{
Antibodies to Bordetella pertussis antigens in maternal and cord blood pairs: a Thai cohort study
}

\author{
Nasamon Wanlapakorn $^{1}$ ， Thanunrat Thongmee ${ }^{1}$, Preeyaporn Vichaiwattana ${ }^{1}$, Elke Leuridan ${ }^{2}$, Sompong \\ Vongpunsawad ${ }^{1}$, Yong Poovorawan Corresp. 1 \\ ${ }^{1}$ Center of Excellence in Clinical Virology, Department of Pediatrics, Faculty of Medicine, Chulalongkorn University, Bangkok, Thailand \\ 2 Centre for the Evaluation of Vaccination, Vaccine \& Infectious Disease Institute, University of Antwerp, Belgium \\ Corresponding Author: Yong Poovorawan \\ Email address: Yong.P@chula.ac.th
}

Background: Pertussis is a vaccine-preventable disease, yet an increasing incidence of pertussis occurs in many countries. Thailand has a long-standing pertussis vaccination policy, therefore most expectant mothers today had received vaccines as children. The resurgence of pertussis among Thai infants in recent years led us to examine the pre-existing antibodies to Bordetella pertussis antigens in a cohort of 90 pregnant women.

Methods: We evaluated the IgG to the Pertussis toxin (PT), filamentous hemagglutinin (FHA) and pertactin (PRN) in maternal and cord blood sera using commercial enzyme-linked immunosorbent assays (ELISA).

Results: When values of $>10 \mathrm{IU} / \mathrm{ml}$ were accepted as potential protective concentrations, we found that the percentages of unprotected infants were $73.3 \%, 43.3 \%$ and $75.5 \%$ for anti-PT, anti-FHA and anti-PRN $\lg G$, respectively.

Discussion: These results may explain the susceptibility for pertussis among newborn infants in Thailand and support the requirement for a pertussis booster vaccine during pregnancy, which may contribute to the passive seroprotection among newborns during the first months of life. 
1 Antibodies to Bordetella pertussis antigens in maternal and cord blood pairs: A Thai cohort 2 study.

3 Nasamon Wanlapakorn ${ }^{1}$, Thanunrat Thongmee ${ }^{1}$, Preeyaporn Vichaiwattana ${ }^{1}$, Elke Leuridan ${ }^{2}$, 4 Sompong Vongpunsawad ${ }^{1}$, Yong Poovorawan ${ }^{1 *}$

$5{ }^{1}$ Center of Excellence in Clinical Virology, Department of Pediatrics, Faculty of Medicine, 6 Chulalongkorn University, Bangkok, Thailand

$7{ }^{2}$ Centre for the Evaluation of Vaccination, Vaccine \& Infectious Disease Institute, University of 8 Antwerp, Belgium

9

10 Corresponding authors: Professor Yong Poovorawan

$11 *$ Correspondence: yong.p@,chula.ac.th 


\section{Abstract}

15 Background: Pertussis is a vaccine-preventable disease, yet an increasing incidence of pertussis

16 occurs in many countries. Thailand has a long-standing pertussis vaccination policy, therefore

17 most expectant mothers today had received vaccines as children. The resurgence of pertussis

18 among Thai infants in recent years led us to examine the pre-existing antibodies to Bordetella

19 pertussis antigens in a cohort of 90 pregnant women.

20 Methods: We evaluated the IgG to the Pertussis toxin (PT), filamentous hemagglutinin (FHA)

21 and pertactin (PRN) in maternal and cord blood sera using commercial enzyme-linked

22 immunosorbent assays (ELISA).

23 Results: When values of $\geq 10 \mathrm{IU} / \mathrm{ml}$ were accepted as potential protective concentrations, we

24 found that the percentages of unprotected infants were $73.3 \%, 43.3 \%$ and $75.5 \%$ for anti-PT, 25 anti-FHA and anti-PRN IgG, respectively.

26 Discussion: These results may explain the susceptibility for pertussis among newborn infants in

27 Thailand and support the requirement for a pertussis booster vaccine during pregnancy, which

28 may contribute to the passive seroprotection among newborns during the first months of life. 


\section{Introduction}

Countries with universal pertussis vaccination have experienced pertussis resurgence in recent years especially those implementing the acellular pertussis (aP) vaccines (Clarke et al., 2013; Octavia et al., 2012; van der Maas et al., 2013; Winter et al., 2014). Recent evidence suggests that this was partly due to the waning immunity as a result of the aP-vaccine use (Klein et al., 2012; Liko et al., 2013). Maternal vaccination during pregnancy is an effective strategy to prevent pertussis-related morbidity and mortality in newborns who are at the highest risk for infection and hospitalization (ACIP, 2013). Vaccination during pregnancy boosts the immune response against $B$. pertussis in expectant mothers and affords the transplacental transfer of antibodies to the baby, thus conferring protection to pertussis in infants during the first few months of life (Hoang et al., 2016; Maertens et al., 2016; Munoz et al., 2014; Vizzotti et al., 2016).

Thailand implemented a routine infant immunization program with two doses of the Diphtheria-Tetanus toxoid-whole-cell Pertussis (DTwP) vaccine beginning in 1977. From 1992 onward, the country's Expanded Program on Immunization (EPI) offers five doses of DTwP to infants at 2, 4, 6, 18 and 48 months of age. A booster dose of Tetanus Toxoid (TT), which has since been replaced by diphtheria-tetanus vaccine (dT) in 2012, is also recommended during adolescence and every 10 years thereafter. Since then, data from the national passive surveillance showed a relatively low burden of pertussis in the general population. Between 2007 and 2014, the incidence of pertussis in Thailand was reportedly 6-25 per year (0.01-0.04 per 100,000 individuals) (Bureau of epidemiology, 2014). In 2015 and 2016, however, there was an increased incidence of 51 cases $(0.08 / 100,000)$ and 72 cases $(0.11 / 100,000)$, respectively (Bureau of epidemiology, 2016). Significant numbers of morbidity belonged to children one 
52 year of age or younger and this age group bears the greatest risk of pertussis morbidity. In

53 addition, these numbers are likely to be under-estimated due to missed pertussis diagnosis and

54 inadequate laboratory confirmation. To date, Thailand has not yet integrated the Tetanus-

55 reduced dose of diphtheria and acellular pertussis (Tdap) booster during adolescence or

56 pregnancy.

57 Despite the universal whole-cell pertussis (wP) vaccination among newborns in Thailand,

58 anti-PT IgG has been shown to wane precipitously (Wanlapakorn et al., 2016). To evaluate the

59 susceptibility to pertussis among infants born to Thai mothers, we aimed to determine the

60 baseline concentration of IgG against PT, FHA and PRN in pregnant women who did not receive

61 pertussis vaccination during pregnancy. Data from this study may be important in providing

62 evidence-based consideration for a pertussis booster during pregnancy.

\section{Materials and Methods}

\section{Study Population}

The study was approved by the Institutional Review Board of the Faculty of Medicine of Chulalongkorn University (IRB No. 154/58). The serum samples were archived residual samples from 90 mother-cord blood pairs collected between July 2011 and August 2012 to examine serological protection against tetanus among pregnant women at King Chulalongkorn Memorial Hospital in Bangkok. All samples were deidentified and anonymous, therefore no consent was required and the permission to use these samples was granted by the Director of King Chulalongkorn Memorial Hospital. Inclusion criteria were healthy pregnant Thai women between 15 and 45 years who sought antenatal care at King Chulalongkorn Memorial Hospital in 2011 and 2012. During their first visit, history of tetanus immunization and blood samples were obtained. Cord blood samples were collected at the time of delivery. None of the mothers in this 
study were able to provide their vaccination records, therefore women born prior to 1977 were assumed to have never received DTwP, while those born after 1977 were assumed to have had between 2 and 5 doses of DTwP.

\section{Antibody measurement}

The anti-PT, anti-FHA and anti-PRN IgG were analyzed quantitatively using commercial ELISA kits (EUROIMMUN, Lübeck, Germany) according to the manufacturer's instructions. The controls of the ELISA kits were calibrated using the first International World Health Organization standards (WHO International Standard Pertussis Antiserum, human, $1^{\text {st }}$ IS NIBSC Code $06 / 140)$ and quantified in international unit per milliliter (IU/ml). The international reference preparation of the Food and Drug Administration (Bethesda, USA) was used. Sera were initially diluted 1:101 for the test and higher dilutions were performed as necessary. The lower limit of quantification (LLOQ) for anti-PT, anti-FHA and anti-PRN IgG is 5 International Unit (IU) per ml of serum. Values below LLOQ were calculated as half of the LLOQ. For anti$\mathrm{PT}$, Values $<5 \mathrm{IU} / \mathrm{ml}$ were interpreted as seronegative, $5-40 \mathrm{IU} / \mathrm{ml}$ as no evidence of recent acute infection, $40-100 \mathrm{IU} / \mathrm{ml}$ as probable past exposure to pertussis, and $>100 \mathrm{IU} / \mathrm{ml}$ as acute pertussis infection or recent vaccination.

\section{Statistical analysis}

The IgG level was expressed as geometric mean concentrations (GMC) with standard error of the mean (SE). Data were analyzed using SPSS software (IBM Inc., Armonk, NY, USA), SigmaPlot (Systat Software, San Jose, CA) and R statistical software. Chi square and Fisher's exact test were used for statistical comparisons of seronegativity rates of pregnant women born before or after the pertussis inclusion in the EPI. Linear regression model was used 
97 to predict antibody levels in cord sera. A simple and multivariable regression models were used

98 to analyze the predictors affecting the antibody level in the cord blood. 


\section{Results}

Recent statistics in Thailand showed an increase in the number of pertussis in the 0 -1 age group (Table 1). Between 2013 and 2015, 3 pertussis-related deaths were reported. To determine whether expectant mothers in recent years possessed any immunity against $B$. pertussis, we tested a cohort of convenient serum samples for anti-PT, anti-FHA, and anti-PRN IgG obtained from mothers and the cord blood, the latter of which served as a surrogate for infant blood samples at birth. The majority of maternal blood samples were obtained during the first trimester of pregnancy as defined by the gestational age (GA) at less than 12 weeks (Table 2). All babies except one were born healthy. One baby born at GA of 23 weeks died hours after birth due to multiple congenital anomalies.

The GMC of anti-PT, anti-FHA and anti-PRN IgG in maternal and cord sera (Fig 1) were derived from individual values (S1 Table). Maternal and cord sera samples demonstrated similar anti-PT and anti-PRN levels, both of which were lower than that of anti-FHA. When values of $>$ $10 \mathrm{IU} / \mathrm{ml}$ were accepted as potential protective concentrations, we found that the percentages of unprotected infants were $73.3 \%, 43.3 \%$ and $75.5 \%$ for anti-PT, anti-FHA and anti-PRN IgG, respectively. Comparison of all 90 mother-infant paired samples showed that maternal anti-PT, anti-FHA and anti-PRN IgG correlated significantly with cord blood values (Fig 2).

Anti-PT IgG levels can reflect not only the circulating antibody from past vaccination but also the recent exposure to pertussis. When the levels were classified as $<5 \mathrm{IU} / \mathrm{ml}$ (seronegative), 5-40 IU/ml (no evidence of recent infection), 40-100 IU/ml (probable past exposure to pertussis) and $>100 \mathrm{IU} / \mathrm{ml}$ (acute or recent infection). We found that $56.7 \%$ of pregnant women were seronegative for anti-PT. Forty percent of the maternal serum samples did not show evidence of recent infection, as defined by antibody levels between 5-40 IU/ml. Three women (aged 19, 28, 
122 and 40 years) possessed anti-PT IgG titers of $>100 \mathrm{IU} / \mathrm{ml}$, which represented $3.3 \%$ of the

123 samples. Two-thirds of these high anti-PT IgG samples also possessed $>350 \mathrm{IU} / \mathrm{ml}$ of anti-PRN

$124 \mathrm{IgG}$, while one-third had $>350 \mathrm{IU} / \mathrm{ml}$ of anti-FHA IgG, suggesting possible recent infection.

125 Pregnant women who were born before the pertussis vaccine was implemented into the

126 EPI program (30/90) were 34 years of age or older (Table 3). There were no differences in the

127 proportion of anti-PT IgG seronegativity or recently infected rates in women born before or after

128 the EPI program.

To determine factors affecting the antibody level in the cord sera, we tested two models.

Using the bivariable model, which assumed that level of anti-PT, anti-FHA and anti-PRN in cord sera was entirely attributed to the maternal sera, we found that antibody level in maternal sera significantly affected the level in cord sera $(\mathrm{p}<0.001)$. This model would account for $88.4 \%$, $89.2 \%$ and $95.9 \%$ of the variations in cord anti-PT, andti-FHA and anti-PRN IgG, respectively.

Using the multivariable model, which took the gestational age at delivery and the interval between maternal and cord sera collection into consideration, neither parameters were significantly associated with the level in cord sera and did not confound the effect of maternal sera.

\section{Discussion}

The increase in the prevalence of reported pertussis morbidity among infants in Thailand cord blood paired samples in order to determine serological baseline. Despite receiving wP vaccination as children, most pregnant women in this study demonstrated low anti-PT, anti-FHA and anti-PRN IgG, which were also reflected by the serology status in the corresponding cord 
144 serum samples. The finding suggests that a significant number of women and their newborn 145 children are susceptible to pertussis. Additionally, the anti-PT seronegative rates were similar

146 irrespective of vaccination status and implicated waning immunity for individuals who had

147 received wP vaccination.

Previous studies examining seroprevalence of anti-PT IgG in pregnant women revealed that maternal GMCs of anti-PT detected at delivery ranged between 2.4-19.39 ELISA Units or IU/ml (de Voer et al., 2009; Ercan et al., 2013; Gonik et al., 2005; Healy et al., 2004; Healy et al., 2006; Heininger et al., 2009; Hoang et al., 2016; Shakib et al., 2010; van den Berg et al., 2010; Villarreal Perez et al., 2017). Although our GMC of $5.18 \mathrm{IU} / \mathrm{ml}$ was towards the low side of this range, it was similar to the values reported from the nationwide seroprevalence survey of anti-PT IgG in 2014 (Wanlapakorn et al., 2016). In that study, the GMCs of anti-PT IgG were 4.53 and $3.76 \mathrm{IU} / \mathrm{ml}$ among $21-30$ and 31-40 year-olds, respectively. This reflects the susceptibility to pertussis among women of child-bearing age in Thailand and supports the potential benefit of a booster during adolescence and pregnancy.

FHA is a cell surface protein found in many bacteria including Bordetella pertussis. It plays a key role in bacterial adhesion to epithelial cells and thus contributes to its pathogenesis. Previous studies have shown that women who did not receive pertussis vaccination during pregnancy had anti-FHA IgG ranging from 6.9 to 26.6 ELISA units or IU/ml (de Voer et al., 2009; Ercan et al., 2013; Gonik et al., 2005; Healy et al., 2004; Heininger et al., 2009; Hoang et al., 2016; van den Berg et al., 2010). Our results were consistent with these reported values. In addition to PT, PRN is another virulence factor, which promotes bacterial adhesion to epithelial cells. Anti-PRN concentrations correlated with protection to pertussis disease as they facilitate phagocytosis by polymorphonuclear cells (Hellwig et al., 2003). The levels of anti-PRN IgG in 
167 this study were consistent with those reported in recent studies, which found that anti-PRN IgG 168 in maternal sera were between 4.09-13.5 ELISA units or IU/ml (de Voer et al., 2009; Gonik et 169 al., 2005; Hoang et al., 2016; van den Berg et al., 2010; Villarreal Perez et al., 2017). IgG levels of $>100 \mathrm{IU} / \mathrm{ml}$, which indicated recent $B$. pertussis infection (Nooitgedagt et al., 2009;

172 Plans et al., 2014; Plans et al., 2008). These women were presumed to be protected from 173 pertussis during pregnancy and were shown to transfer anti-PT IgG to their fetus. With the 174 assumption of vaccination based on age, but without available vaccination record or clinical 175 confirmation of pertussis, we concluded that $3.3 \%$ of mothers with anti-PT IgG titer $>100 \mathrm{IU} / \mathrm{ml}$ in this study had recently been exposed to B. pertussis. For $30-40 \%$ of pregnant women who had anti-PT IgG titers between 5-40 IU/ml, we cannot eliminate the possibility of waning immunity associated with $B$. pertussis exposure sometime in the past few years. If this was indeed representative of the whole population, then it is plausible that B. pertussis exposure is not uncommon despite universal childhood vaccination in Thailand. transport, which result in the increased level of antibodies in the cord blood at term when compared to maternal sera (Ercan et al., 2013; Hardy-Fairbanks et al., 2013; Heininger et al., 2009). During early infancy, therefore, protection from diseases often relies on the passively transferred maternal antibodies to newborn infants. In this study, we demonstrated that maternal anti-PT, anti-FHA and anti-PRN IgG levels highly correlated with those of the paired cord blood.

187 We demonstrated that the majority of Thai infants were unlikely to be protected from pertussis regardless of the mothers' vaccination status. Although the increase in reported pertussis incidence seen in very young children may reflect better disease awareness by clinicians, it may 
190 also reflect the under-diagnosis of pertussis in adults and the waning of the immunity to $B$.

191 pertussis in the general population.

192 Future work to assess the effect of a booster dose of pertussis vaccination among

193 pregnant women in terms of antibody to $B$. pertussis antigens, the extent of passive maternal-

194 fetal antibody transfer, and long term immunity in Thai infants induced by whole cell or acellular

195 pertussis vaccine is ongoing. To reduce the risk of pertussis-related complications and infant

196 mortality in countries using wP vaccine in their EPI program, further studies to examine the

197 effectiveness of Tdap vaccination during pregnancy, the long-term effect of Tdap in wP-

198 vaccinated infants, and possible interference of maternal-derived antibody with wP-vaccinated

199 infants will be important.

\section{Conclusions}

201 Our results explained the susceptibility for pertussis among newborn infants in Thailand

202 and supported the requirement for a pertussis booster vaccine during pregnancy. Maternal

203 vaccination during pregnancy may provide passive seroprotection in newborns during the first

204 months of life.

205 Acknowledgements

206 The authors would like to thank Professor Teerapong Tantawichien in the Department of

207 Medicine (Faculty of Medicine, Chulalongkorn University) for providing serum samples from

208 the project that aimed to study tetanus serological protection among pregnant woman at King

209 Chulalongkorn Memorial Hospital. We also would like to thank the staff at the Center of

210 Excellence in Clinical Virology and King Chulalongkorn Memorial Hospital for technical

211 assistance. 


\section{References}

213 Advisory Committee on Immunization Practices (ACIP). Updated recommendations for use of tetanus toxoid, reduced diphtheria toxoid, and acellular pertussis vaccine (Tdap) in pregnant women. 2013. Morbidity and Mortality Weekly Report 62:131-135.

216

217

218

219

220

221

222

223

224

225

226

227

228

229

230

231

232

233

Bureau of epidemiology, Ministry of Public Health, Thailand. Summaries of selected notifiable diseases from the annual epidemiology surveillance report. 2014. Available at http://www.boe.moph.go.th/Annual/AESR2014/aesr2557/Part\%201/1-3/pertussis.pdf (assessed 5 January 2017)

Bureau of epidemiology, Ministry of Public Health, Thailand. Incidence of communicable diseases (Pertussis). 2016. Available at http://www.boe.moph.go.th/boedb/surdata/index.php (assessed 7 February 2017)

Clarke MF, Rasiah K, Copland J, Watson M, Koehler AP, Dowling K, and Marshall HS. 2013. The pertussis epidemic: informing strategies for prevention of severe disease. Epidemiology and Infection 141:463-471. DOI: 10.1017/s095026881200091x de Voer RM, van der Klis FR, Nooitgedagt JE, Versteegh FG, van Huisseling JC, van Rooijen DM, Sanders EA, and Berbers GA. 2009. Seroprevalence and placental transportation of maternal antibodies specific for Neisseria meningitidis serogroup C, Haemophilus influenzae type B, diphtheria, tetanus, and pertussis. Clinical Infectious Diseases 49:5864. DOI: $10.1086 / 599347$

Ercan TE, Sonmez C, Vural M, Erginoz E, Torunoglu MA, and Perk Y. 2013. Seroprevalance of pertussis antibodies in maternal and cord blood of preterm and term infants. Vaccine 31:4172-4176. DOI: 10.1016/j.vaccine.2013.06.088 
234 Gonik B, Puder KS, Gonik N, and Kruger M. 2005. Seroprevalence of Bordetella pertussis

235

236

237

238

239

240

241

242

243

244

245

246

247

248

249

250

251

252

253

254

255 antibodies in mothers and their newborn infants. Infectious Diseases in Obstetrics Gynecology 13:59-61. DOI: 10.1080/10647440500068289

Hardy-Fairbanks AJ, Pan SJ, Decker MD, Johnson DR, Greenberg DP, Kirkland KB, Talbot EA, and Bernstein HH. 2013. Immune responses in infants whose mothers received Tdap vaccine during pregnancy. The Pediatric Infectious Disease Journal 32:1257-1260. DOI: 10.1097/INF.0b013e3182a09b6a

Healy CM, Munoz FM, Rench MA, Halasa NB, Edwards KM, and Baker CJ. 2004. Prevalence of pertussis antibodies in maternal delivery, cord, and infant serum. The Journal of Infectious Diseases 190:335-340. DOI: 10.1086/421033

Healy CM, Rench MA, Edwards KM, and Baker CJ. 2006. Pertussis serostatus among neonates born to Hispanic women. Clinical Infectious Diseases 42:1439-1442. DOI: $10.1086 / 503567$

Heininger U, Riffelmann M, Leineweber B, and Wirsing von Koenig CH. 2009. Maternally derived antibodies against Bordetella pertussis antigens pertussis toxin and filamentous hemagglutinin in preterm and full term newborns. The Pediatric Infectious Disease Journal 28:443-445. DOI: 10.1097/INF.0b013e318193ead7

Hellwig SM, Rodriguez ME, Berbers GA, van de Winkel JG, and Mooi FR. 2003. Crucial role of antibodies to pertactin in Bordetella pertussis immunity. The Journal of Infectious Diseases 188:738-742. DOI: $10.1086 / 377283$

Hoang HT, Leuridan E, Maertens K, Nguyen TD, Hens N, Vu NH, Cabore RN, Duong HT, Huygen K, Van Damme P, and Dang AD. 2016. Pertussis vaccination during pregnancy 
in Vietnam: Results of a randomized controlled trial Pertussis vaccination during pregnancy. Vaccine 34:151-159. DOI: 10.1016/j.vaccine.2015.10.098

258

259

260

261

262

263

264

265

266

267

Klein NP, Bartlett J, Rowhani-Rahbar A, Fireman B, and Baxter R. 2012. Waning protection after fifth dose of acellular pertussis vaccine in children. The New England Journal of Medicine 367:1012-1019. DOI: 10.1056/NEJMoa1200850

Liko J, Robison SG, and Cieslak PR. 2013. Priming with whole-cell versus acellular pertussis vaccine. The New England Journal of Medicine 368:581-582. DOI: 10.1056/NEJMc1212006

Maertens K, Cabore RN, Huygen K, Vermeiren S, Hens N, Van Damme P, and Leuridan E. 2016. Pertussis vaccination during pregnancy in Belgium: Follow-up of infants until 1 month after the fourth infant pertussis vaccination at 15 months of age. Vaccine 34:36133619. DOI: $10.1016 /$ j.vaccine.2016.04.066

Munoz FM, Bond NH, Maccato M, Pinell P, Hammill HA, Swamy GK, Walter EB, Jackson LA, Englund JA, Edwards MS, Healy CM, Petrie CR, Ferreira J, Goll JB, and Baker CJ. 2014. Safety and immunogenicity of tetanus diphtheria and acellular pertussis (Tdap) immunization during pregnancy in mothers and infants: a randomized clinical trial. JAMA 311:1760-1769. DOI: 10.1001/jama.2014.3633

Nooitgedagt JE, de Greeff SC, Elvers BH, de Melker HE, Notermans DW, van Huisseling H, and Versteegh FG. 2009. Seroprevalence of Bordetella pertussis infection during pregnancy measured by IgG antibodies against pertussis toxin. Clinical Infectious Diseases 49:1086-1089. DOI: 10.1086/605575

Octavia S, Sintchenko V, Gilbert GL, Lawrence A, Keil AD, Hogg G, and Lan R. 2012. Newly emerging clones of Bordetella pertussis carrying prn2 and ptxP3 alleles implicated in 
Australian pertussis epidemic in 2008-2010. The Journal of Infectious Diseases 205:1220-1224. DOI: 10.1093/infdis/jis 178

Plans P, Alvarez E, de Ory F, Campins M, Paya T, Balfagon P, Godoy P, Cayla J, Carreras R, Cabero L, and Dominguez A. 2014. Prevalence of antibody to Bordetella pertussis in neonates and prevalence of recent pertussis infection in pregnant women in Catalonia (Spain) in 2003 and 2013. The Pediatric Infectious Disease Journal 33:1114-1118. DOI: 10.1097/inf.0000000000000413

Plans P, Jansa J, Doshi N, Harrison TG, and Plasencia A. 2008. Prevalence of pertussis antibodies in umbilical cord blood samples in Catalonia, Spain. The Pediatric Infectious Disease Journal 27:1023-1025.

Shakib JH, Ralston S, Raissy HH, Stoddard GJ, Edwards KM, and Byington CL. 2010. Pertussis antibodies in postpartum women and their newborns. Journal of Perinatology 30:93-97. DOI: $10.1038 /$ jp.2009.138

van den Berg JP, Westerbeek EA, Berbers GA, van Gageldonk PG, van der Klis FR, and van Elburg RM. 2010. Transplacental transport of IgG antibodies specific for pertussis, diphtheria, tetanus, haemophilus influenzae type $b$, and Neisseria meningitidis serogroup $\mathrm{C}$ is lower in preterm compared with term infants. The Pediatric Infectious Disease Journal 29:801-805.

van der Maas NA, Mooi FR, de Greeff SC, Berbers GA, Spaendonck MA, and de Melker HE. 2013. Pertussis in the Netherlands, is the current vaccination strategy sufficient to reduce disease burden in young infants? Vaccine 31:4541-4547. DOI: 10.1016/j.vaccine.2013.07.060 
301 Villarreal Perez JZ, Ramirez Aranda JM, de la OCM, Zamudio Osuna MJ, Perales Davila J, 302 Ballesteros Elizondo MR, Gomez Meza MV, Garcia Elizondo FJ, and Rodriguez

303

304

305

306

307

308

309

310

311

312

313

314

315

316

317
Gonzalez AM. 2017. Randomized clinical trial of the safety and immunogenicity of the Tdap vaccine in pregnant Mexican women. Human Vaccines and Immunotherapeutics 13:128-135. DOI: $10.1080 / 21645515.2016 .1232786$

Vizzotti C, Juarez MV, Bergel E, Romanin V, Califano G, Sagradini S, Rancano C, Aquino A, Libster R, Polack FP, and Manzur J. 2016. Impact of a maternal immunization program against pertussis in a developing country. Vaccine 34:6223-6228. DOI:

10.1016/j.vaccine.2016.10.081

Wanlapakorn N, Ngaovithunvong V, Thongmee T, Vichaiwattana P, Vongpunsawad S, and Poovorawan Y. 2016. Seroprevalence of Antibodies to Pertussis Toxin among Different Age Groups in Thailand after 37 Years of Universal Whole-Cell Pertussis Vaccination. PLoS One 11:e0148338. DOI: 10.1371/journal.pone.0148338

Winter K, Glaser C, Watt J, and Harriman K. 2014. Pertussis epidemic--California, 2014. Morbidity and Mortality Weekly Report 63:1129-1132. 
Figure 1

Simple linear regression model of $\lg G$ to PT (A), FHA (B) and PRN (C) in maternal and cord sera.

$\mathrm{R}^{2}$ for anti-PT $=0.854, \mathrm{p}$ value $<0.001, \mathrm{R}^{2}$ for anti-FHA $=0.894, \mathrm{p}$ value $<0.001, \mathrm{R}^{2}$ for anti-PRN $=0.913, p$-value $<0.001$.

A

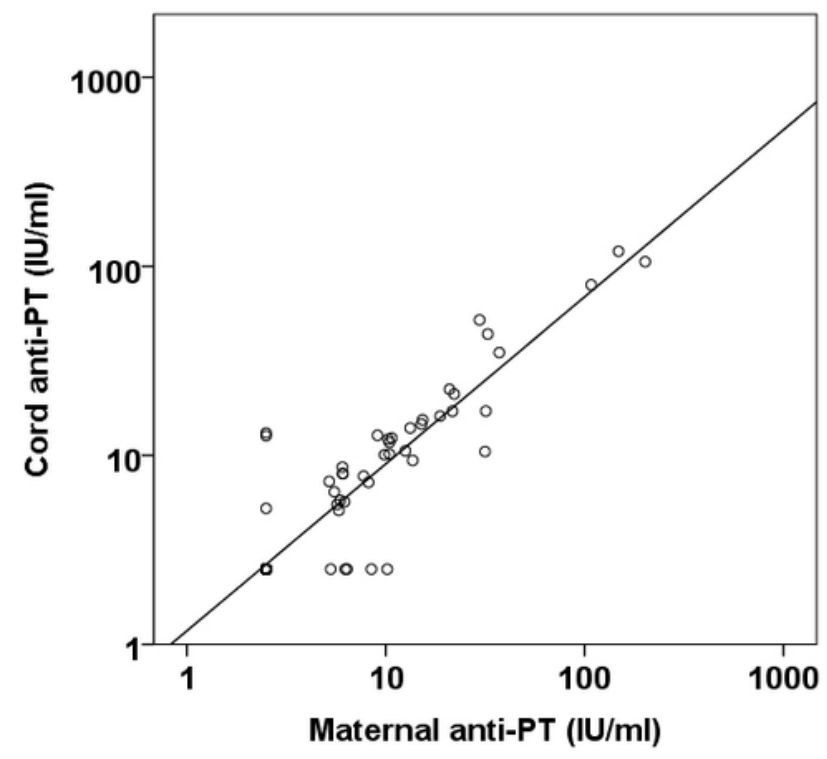

B

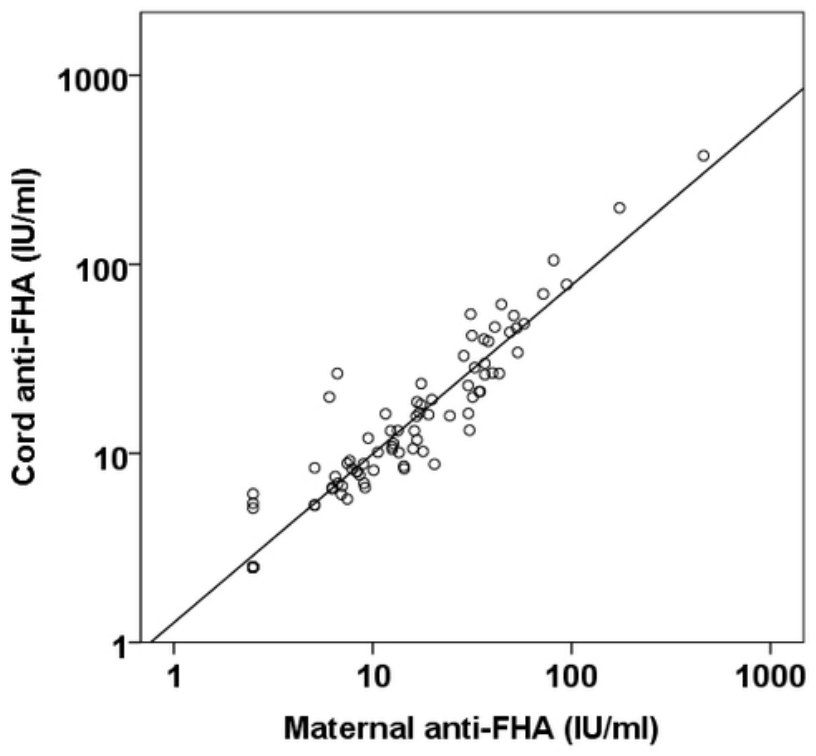

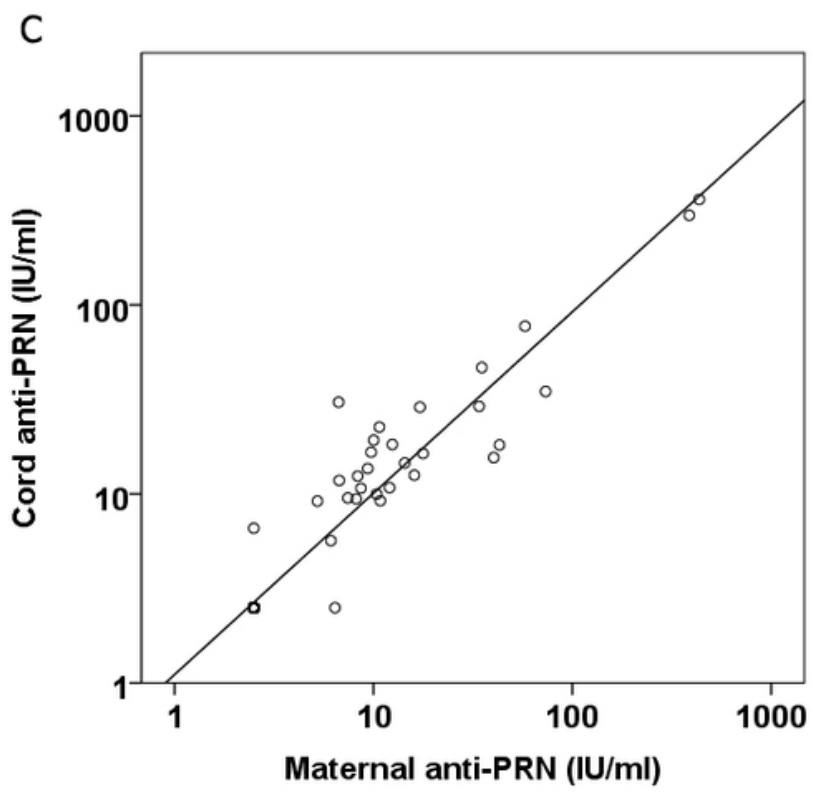




\section{Table $\mathbf{1}$ (on next page)}

Pertussis incidence in Thailand between 2011 and 2016 by age group.

Data were retrieved from the annual epidemiology surveillance report by the Bureau of Epidemiology, Department of Disease Control, Ministry of Public Health, Thailand. The numbers represented suspected, probable and confirmed cases* reported annually. 
1 Table 1. Pertussis incidence in Thailand between 2011 and 2016 by age group. Data were retrieved from the annual

2 epidemiology surveillance report by the Bureau of Epidemiology, Department of Disease Control, Ministry of Public Health,

3 Thailand. The numbers represented suspected, probable and confirmed cases* reported annually.

\begin{tabular}{|c|c|c|c|c|c|c|c|c|c|c|}
\hline $\begin{array}{c}\text { Age group } \\
\text { (years) }\end{array}$ & $0-1$ & $1-4$ & $5-9$ & $10-14$ & $15-24$ & $25-34$ & $35-44$ & $45-54$ & $55-64$ & 65 and above \\
\hline Year & & & & & & & & & & \\
\hline 2016 & 27 & 16 & 9 & 4 & 3 & 5 & 3 & 0 & 0 & 5 \\
\hline 2015 & 35 & 8 & 3 & 0 & 0 & 1 & 0 & 1 & 2 & 1 \\
\hline 2014 & 11 & 1 & 1 & 1 & 0 & 1 & 0 & 0 & 0 & 1 \\
\hline 2013 & 11 & 4 & 1 & 1 & 0 & 1 & 2 & 2 & 1 & 1 \\
\hline 2012 & 6 & 2 & 3 & 3 & 1 & 0 & 1 & 1 & 0 & 0 \\
\hline 2011 & 7 & 2 & 1 & 1 & 0 & 0 & 1 & 0 & 0 & 0 \\
\hline
\end{tabular}

4 *Pertussis case definitions; A suspected case is a patient presenting with a cough illness lasting $\geq 2$ weeks with at least one of the

5 following signs or symptoms: paroxysms of coughing; or inspiratory "whoop"; or post-tussive vomiting. A probable case is defined as

6 a suspected case with epidemiologic linkage to a laboratory-confirmed case. A confirmed case is defined as a suspected case with

7 laboratory confirmation by polymerase chain reaction or bacterial culture. 


\section{Table 2 (on next page)}

Demographic characteristics of pregnant women and infants in the study.

Data were presented as mean and range. 
1 Table 2. Demographic characteristics of pregnant women and infants in the study. Data

2 were presented as mean and range.

\begin{tabular}{|l|c|}
\hline Characteristics & \\
\hline Nunmber of pregnant women & $30.9(19-42)$ \\
\hline Mean age in years (range) & $65(72 \%)$ \\
\hline No. of participants whose maternal sera were collected during & $24(27 \%)$ \\
- Second trimester (GA13-28 weeks) & $1(1 \%)$ \\
\hline Third trimester (GA $\geq 29$ weeks) & $11(5-29)$ \\
\hline Mean GA at maternal blood collection in weeks (range) & $37.6(23-40)$ \\
\hline Mean GA at delivery in weeks (range) & $2,952(980-4,060)$ \\
\hline Infant birth weight in grams (range) & $11.1 \%$ \\
\hline Percentage of premature delivery (GA $<37$ weeks) & $3.3 \%$ \\
\hline Percentage of twins & \\
\hline
\end{tabular}

3 


\section{Figure 2}

Maternal and cord anti-PT, anti-FHA and anti-PRN IgG in this study.

Values are expressed as geometric mean concentration (GMC) in IU/ml. Error bars indicated the standard error (SE) of the mean. Mean + SE (IU/ml) for each concentration were: anti-PT in maternal sera $(5.19+1.11)$, anti-PT in cord sera $(5.05+1.11)$, anti-FHA in maternal sera $(13.10+1.13)$, anti-FHA in cord sera $(12.64+1.12)$, anti-PRN in maternal sera $(4.65+1.12)$, and anti-PRN in cord sera $(4.84+1.12)$.

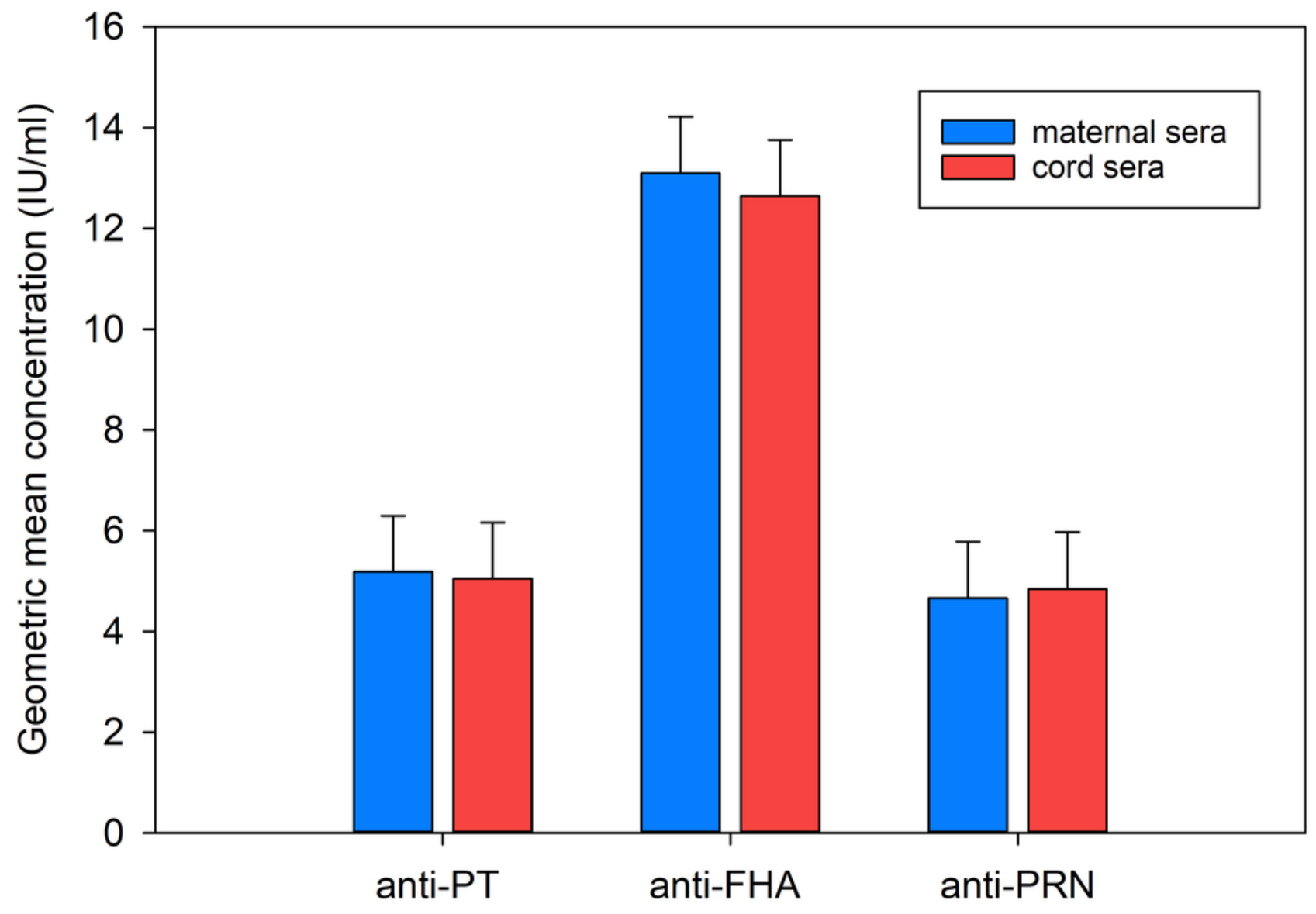




\section{Table 3 (on next page)}

Anti-PT IgG in pregnant women born before or after the implementation of pertussis vaccination.

There were no differences in the proportion of seronegativity or recently infected rates in women born before or after the EPI program. 
1 Table 3. Anti-PT IgG in pregnant women born before or after the implementation of 2 pertussis vaccination. There were no differences in the proportion of seronegativity or recently 3 infected rates in women born before or after the EPI program.

\begin{tabular}{|c|c|c|c|}
\hline $\begin{array}{c}\text { Anti-PT IgG } \\
(\mathrm{IU} / \mathrm{ml})\end{array}$ & Before EPI & After EPI & $p$-value \\
& $($ aged $\geq 34$ years $)(\mathrm{n}=30)$ & $($ aged $<34$ years $)(\mathrm{n}=60)$ & \\
\hline$<5$ & 16 & 35 & 0.660 \\
\hline $5-40$ & 13 & 23 & 0.656 \\
\hline $40-100$ & 0 & 0 & - \\
\hline$>100$ & 1 & 2 & N/A \\
\hline
\end{tabular}

4 N/A; Not Applicable 